\title{
Exploration and Prediction of Crime Data Through Supervised Machine Learning Algorithms
}

\author{
Shruti ${ }^{1}$ and Rajesh Kumar Singh ${ }^{2}$ \\ ${ }^{1} H M R$ Institute of Technology and Management, Hamidpur, Delhi, India \\ ${ }^{2}$ SUS Institute of Computer, Tangori, India
}

\section{ABSTRACT}

The technique of obtaining meaningful information or knowledge from vast data sources is known as machine learning. Large quantity of information is gathered throughout criminal investigation process and only valuable information is necessary for analysis. So, Machine Learning may be employed for this purpose. Selection of certain Machine Learning approach has larger effect on the outcomes achieved. This is major rationale for the performance comparison and selection of top performing Machine Learning algorithm. Classifying instances using a Decision Tree is a basic notion that anybody can comprehend. It is a Supervised Machine Learning as Decision-Tree-Classifier (DTC) in which the data is segregated according to a particular parameter and supervision is continually performed. Machine learning has only recently begun to find greater adoption in both research and practise, and as a consequence, the bulk of current implementations employ data mining and Decision-Tree-Classifier (DTC) approaches. These specific computations have an accuracy of over 43 percent, which is very excellent when consider that are judging crimes from massive data and places.

\section{KEY WORDS: CRIME DATA, SUPERVISED MACHINE LEARNING ALGORITHMS, DECISION-TREE-CLASSIFIER (DTC).}

\section{INTRODUCTION}

Citizens in most nations have a duty to alert the police of crimes in progress, but some forms of crime may be handled by specialised law enforcement authorities. Crimes are usually investigated at three distinct phases: when the first discovery that a crime has occurred, when a suspect is identified, and before evidence is adequate to accuse the culprit in court. The majority of criminal incidents are reported by citizens and not law enforcement personnel (e.g., victims or witnesses). If there is no recognisable victim, such as obscenity, then crimes involving subjects' agreement (e.g., trading in illegal substances or prostitution) and those without, such as murder, are less likely to be uncovered unless the authorities investigate.

Controversial measures are sometimes necessary in order to discover crimes of this kind. Law enforcement authorities have access to a wide variety of different

Biosc Biotech Res Comm P-ISSN: 0974-6455 E-ISSN: 2321-4007

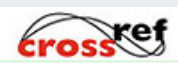

Identifiers and Pagination

Year: 2021 Vol: 14 No (5) Special Issue

Pages: $314-323$

This is an open access article under Creative

Commons License Attribn 4.0 Intl (CC-BY).

DOI: http://dx.doi.org/10.21786/bbrc/14.5/55 scientific tools to help them locate or identify suspects, as well as to further establish the link between the suspect and the crime. Since the late 1980s, DNA fingerprinting of biological evidence (e.g., hair, sperm, and blood) has been possible to dismiss an individual as a suspect with $100 \%$ certainty or be a substantial evidence method of determining if a person is guilty. Paper, glass, paint, and other substances are good sources of information when analysed using microscopic or chemical methods. Certain fibres that were detected on the victim or on the site of the crime might be examined to find if they resemble fibres from the suspect's clothes. If it's shown that the paper on which the document was written was created using an obsolete manufacturing process, the document might be considered a forgery. However, the amount of time and money it takes to create massive databases like these is something to keep in research.

1.1 Suspect identification: A criminal's modus operandi, or technique, may sometimes aid to identify them, since many criminals follow a similar routine in committing their acts. The techniques used by burglars to enter a home, as well as the sort of stuff taken, or the sort of fraud used, may all point to the perpetrator of a crime. When it comes to visual identification of a stranger, the victim is almost always able to do so as well. Victims or witnesses who feel they identify the perpetrator are often shown an album comprising several mug shots of

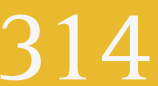


various offenders. The identity of a suspect determined in this method is often requested to take part in a lineup, when witnesses are shown with individuals with comparable traits.

The witness is then asked to choose the culprit. Although academics have long recognised that eyewitnesses are often inaccurate, they didn't necessarily expect many of the false convictions that occurred were because of inaccurate eyewitness identifications (Arunima Kumar, Raju Gopal, 2015; Bachhety et al., 2018). In that people of one race may have trouble recognising those of another race, scholars believe that cross-racial identification leads to mistaken identification. Additionally, following the occurrence, post-incident assimilation is critical because witnesses might radically shift their impression of the offender based on new information. When there is an attempt to commit a crime, the tension associated with it, plus the presence of a weapon, all reduce the trustworthiness of eyewitnesses. Also, such academics have expressed worry that criminal-justice authorities may use normal eyewitness identification methods to influence witness identification so as to maximise the chances of a certain suspect being identified.

However, in the past, authorities in the criminal justice system were mostly resistant to enacting changes that would raise the accuracy of identifications, since such measures would result in fewer identifications overall. As DNA evidence began to be used more effectively in the late 1990s, there was increased attention to instances where eyewitness misidentification had led to a wrongful conviction, including death penalty cases. During the early 21st century, law enforcement agencies were using practises that had been recommended by eyewitness researchers. For these procedures, witness advice to people who are unsure should be avoided, but instead, witnesses should be informed about the possibility of errors and/or made aware of all possible non-suspects in the line-up who might be a good candidate for identification. The line-up should be conducted by a neutral third party who is unaware of any details about the suspects.

1.2 Gathering evidence: Convincing the judge or jury is very vital in nations where the rule of law is firmly established, and this can only be done by gathering sufficient legally acceptable evidence to prove guilt. because the police have very clear knowledge that a certain person is accountable for a crime, but legal proof is lacking to demonstrate guilt (Chung-Hsien et al., 2011). In order to ensure they have all the required evidence; the police use a wide range of investigative tools and methods. In the course of an investigation, it is vital to search a suspect's person or property. Only if there is "probable cause" or "reasonable basis for suspecting" evidence discovered may a search be carried out under the laws of most common-law countries.

If a police officer approaches and says that he or she wants to conduct a search, it is permissible unless the officer refuses to identify himself or herself and has no acceptable basis for the search. A person randomly stopped on the street in the United States may be patted down for a firearm even if there is no indication that they are armed. In most cases, searching a private residence requires the issuing of a search warrant by a court or magistrate. The law only allows for a search warrant to be obtained if the authorities have solid grounds to believe that the precise evidence sought will be located on the premises. Under typical circumstances, a warrant restricts the scope of the search to one location. Because of the use of a search warrant, items collected during an investigation are often kept by the police for use as evidence in any following trial.

1.3 Crime and its Pattern: Crime is a universal social fact that exists in all civilizations at all times. Regardless, similar themes and patterns seem to persist regardless of time and location. Rural and agricultural civilizations with little economic growth. They may be openly condoned and even justified (David \&t Suruliandi, 2017; Hussein et al., 2020; Hussein, 2019). Societies as they modernise and grow more economically sophisticated are unable to tolerate violent actions since these actions become infrequent. The most valuable property often includes items like land and animals, and hence there is less property crime. Because of economic progress, valuable, portable commodities proliferate, and as a result, there is an explosion in theft of such commodities, coupled with an exponential growth in methods of theft. Criminologists have also investigated different police techniques to determine their efficacy.

A significant research study found that arresting the perpetrator often reduces future violence, but has the opposite effect in many instances. A significant amount of research had a significant impact on the way domestic violence is handled in many police agencies. Criminologists have also begun to focus on interventions that aim to lower the chances that particular groups of individuals become criminals (Jain et al. 2020; Hussein \& Croock, 2019). Many elements within biology, psychology, and society enhance the probability of antisocial behaviour, and a focus on long-term crime reduction via social policy that incorporates these variables has been shown effective. A good example is several effective programmes focused on high-risk groups, such as low-income or unmarried moms, who provide pregnant women with prenatal care, postpartum home visits by nurses, and early childhood education for their toddlers. As adolescents, these interventions have been shown to minimise delinquent behaviour (Jayapratha et al., 2018; Mittal et al., 2018). Research has shown that providing preschool education to high-risk youngsters reduces the likelihood that they would offend as adolescents and adults. Criminological research is more likely to be relevant to the establishment of future crime policies, given the increased scrutiny of these programmes by the research community.

\subsection{Types of Crime}

1.4.1 Fraud Detection: The word "fraud" is often used to describe misdirecting or taking unfair advantage of 
someone. Any illegal, deceptive, or misleading conduct, omission, or concealment (whether or not a violation of an obligation or trust) is considered a fraud. Check fraud, online selling, insurance fraud, and credit card fraud are some of the several sorts of fraud. Check fraud is issuance of a check when enough money is not there in an account; Internet sale fraud means selling fake items; Insurance fraud is claiming fake insurance for damage to a vehicle, medical bills, and other purchases (Baesens et al., 2021).

1.4.2 Traffic violence: Vehicle operation is governed by laws, which lead to traffic infractions. As the number of automobiles in cities increases, so does the volume of traffic, and this, along with an increase in traffic offences, produces more severe property damage and increased risk of injury or death for the people who use the roads. Traffic infraction detection technologies are essential to avert such problems (Beland \& Brent, 2018).

1.4.3 Sexual assault: When it comes to committing a criminal attack, it's not whether or not contact is really made, but whether or not the target knows about the threat. There are several types of sexual attacks, including:

Simple Sexual Assault: This consists of physically restraining someone and making them engage in any sexual activity without explicit consent. When it includes the utilisation or threat of the utilisation of a weapon or injury to an outsider, it is known as sexual assault with a weapon.

Aggravated Sexual Assault: This is when a victim sustains bodily harm, such as being ripped apart, mutilated, severely beaten, or near death from a sexual assault. Non-physical, oral ambush resulting in mental, and furthermore mental harm.

II. Literature Review: Singh et al. (2019), presented novel methodology to collect the eyewitness's visual perception as a symbolic representation, in addition to mugshot detection. It enables the detection of criminal physiognomy and face traits. Symbolic representations are modelled via an approach which utilises rough set theory. Through this methodology, they get an instinctive understanding of how criminal minds absorb incomplete and inaccurate information. To check whether our suggested model was right, they utilised a mug picture dataset comprising of 300 offenders faces from the Chinese University of Hong Kong (CUHK). For the visual representation of the 300 faces of criminal CUHK, they used the services of 105 students from the Indian Institute of Information Technology, Allahabad, who were considered eyewitnesses. To prove the theory, the researcher used two modes that are similar to forensic drawings.

Hussein et al. (2020), offer two different hybridization strategies in this work. First, they integrate the Viterbi algorithm with Baum Welch for crime prediction, which leads to the creation of the Viterbi-Baum-Welch hybrid algorithm. In this second example, the technique considered here is a decision tree (DT) augmented with the Viterbi algorithm, which is used to determine the criminal identities of individuals found in the Iraq and India crime dataset. Their prior work is used as a basis for this project. The primary objective is to help improve the outcomes of the model, and getting an accurate model is a side effect. The findings demonstrated both objectives had been achieved in an effective manner.

Jain et al. (2020), Today, all contemporary cities confront a significant criminal issue on their highways. Criminals often turn to road transport as a means of getting away. Due to the absence of proof, theft and other crimes remain unsolved. Tracking cars and criminals still needs a lot of resources to be effective. They come up with a viable machine learning-based criminal detection system employing text and facial recognition algorithms to foil the aforementioned problem. Once these technologies are shown to be effective at parking lots, toll stations, airports, border crossings, etc., they widely used. The text recognition technology uses the characters that appear on Indian number plates to extract the characters and use this as the prediction. At the same time, facial recognition has the capability of performing face recognition on select face areas and mapping the facial features associated with criminals to a database. The research presented in this paper features a proposed system that targets to deliver improvised outcomes to be successful in the majority of cases, even with extreme time constraints and accuracy. Also, it is designed to deliver the successful detection of crime, even under normal working conditions, with machine learning algorithms such as KNN, SVM, and face detection classifiers.

Tayal et al. (2015), developed a collection of tools called data extraction, which is comprised of many modules. They call these modules "tools," and they include data pre-processing, clustering, Google map representation, classification, and WEKA implementation. DE's first module, throughout the time period of 2000-2012, pulls numerous crime datasets from criminal Web sources and organises the data into structured files. The second module, which DP calls DP Cleans, Integrates, and Reduces Crime Data, is creating structured 5,038 crime occurrences. They use 35 present criminal characteristics to represent each of these situations. To ensure that the criminal database is easily accessible, safeguard procedures are adopted. The process of generating two crime groups based on comparable criminal characteristics is investigated using $\mathrm{k}$-means clustering. $\mathrm{K}$-means is helped by Google Maps, which enhances visualisation. Using KNN classification, criminal identification and prediction are studied. The outcomes of our study are verified using WEKA. WEKA certifies the development of two criminal clusters with an accuracy of 93.62 and 93.99 percent. This is an example of our way of making society better. It assists in the solving of crimes and the identification of offenders, helping to reduce crime rates.

Mittal et al. (2019), illustrate the patterns of crime in India change according to population growth and 
urban development. According to authors, the increase in crime incidents, particularly those against women, children, and those in poorer socioeconomic statuses, is a cause for concern for the Indian government. For a better understanding of crimes in India, there is an official database known as the National Crime Records Bureau (NCRB) as well as an application known as Crime Criminal Information System (CCIS) that can be used to look up information and create reports on crimes. Criminals thrive in an environment of chaos and disorder; thus, the police have to comb through extensive amounts of crime data in order to identify contributing elements. Thus, methods are required that can swiftly and effectively identify the elements that influence crime. Because of this, the discipline of machine learning has arisen in the last few years. An analysis of the impacts of the economic crisis on criminal activity in India are being studied in this article, which utilises several machine learning approaches on crime data. For several Indian states, the study has tracked the impact of unemployment rates and GDP on theft, robbery, and burglary. Additionally, Granger causality has been computed between crime rates and economic indices. Finally, it is concluded that a rise in unemployment results in an increase in crime, hence the only way to manage crime is to increase job possibilities.

Prabakaran \& Mitra (2018), introduced the data mining is the discipline concerned with discovering designs or patterns in a large dataset. This is done using processes that include machine learning and database techniques converging to create one system. In addition to the many sectors listed above, it may be used in almost any industry, such as future healthcare, market basket research, education, manufacturing engineering, and criminal investigation. With respect to this, crime investigation is a somewhat intriguing application in which criminal characteristics are used to improve the quality of life for the community. A study compiled this information from numerous data mining methods found in this sector. This work may lead to more efficient crimeprediction and analysis systems.

Qayyum \& Dar (2018), presented the data mining methods to detecting links among data, and machine learning and artificial intelligence methods are very useful when applied to massive datasets. One of the popular areas in data mining nowadays is crime detection, which has distinct patterns of criminology uncovered. It consists of many stages, beginning with criminal characterisation until pattern detection. Various criminal detection strategies have been covered in the literature for this purpose. The approaches utilise in this study are useful for crime detection in general, and they have decided to employ well accepted data mining approaches to fulfil this goal. The research is provided with a tabulation of the different techniques' strengths and weaknesses. This methodology is only for specialised usages, such as identifying the social relationships and roles of criminals in any network. The survey would be a resource for criminologists to locate cutting-edge crime detection strategies that use data mining and a list of both advantages and disadvantages.

Dutta et al. (2017), presented the identity crime is defined as crimes in which someone disguises their identity, therefore obtaining sensitive information about the subject, and as such, it is a sort of identity theft. In this study, credit card application-related identity crime is mostly discussed. In order to overcome the limitations of the current non-data-mining approaches for identity theft, a new data-mining layer of defence has been developed. Communal Detection and Spike Detection methods are used in this unique technique for identifying frauds in apps.

Pavithra \& Suresh (2019), presented an article on fingerprints. They found at the crime site are valuable investigative information. The proposed fingerprint recognition system contains a deep machine learning and Convolutional Neural Network, a kind of deep learning most often used in areas like natural language processing (CNN). The images are collected from crime scenes using a variety of ways, such as advanced photographic technology and chemical and physical processing procedures. Afterward, they are stored in the database. The photographs taken from the crime scene are often fragmentary, and therefore it is difficult to group them into different categories. Prior to processing, fingerprint pictures must first be processed using suitable enhancement techniques. The minutiae (the little, seemingly insignificant details) are taken from the fingerprint scans. Pre-processed data's features are used as inputs into the CNN for training and testing the network. 80\% accuracy in the criminal database was shown utilising experimental database software.

Srivastava et al. (2013), illustrated that the Crime scene investigators which rely on stains for numerous kinds of criminal investigations, including rape, murder, theft, and burglary. Crime scenes often yield stains including blood, semen, saliva, and lipstick. The evidence of lipstick might be detected on clothes, paper, cigarette butts, skin, or anything else. For the criminal identification, questioned lipstick stains might be compared with the suspected one. In the current investigation, Thin Layer Chromatography (TLC) has been used to identify the Lipstick stains. Hussein et al. (2019), In crime detection, data-mining approaches, which may be improved using a variety of methodologies, are used. Classifying and improving the structure is done using the clever way with this work, the decision tree algorithm. This approach is used to compare two datasets: criminals in Iraq and India. The proposal's purpose is to detect offenders via mining utilising an approach that incorporates smart search. Through adjusting the size of the tree, they get greater results than those obtained by standard mining techniques.

Karve et al. (2019), introduced the HOG Face recognition. The purpose of this article is to help the police department help criminals better identify themselves start the 
recognition process by utilising the sketch and visual features that they have provided to generate photos of their faces. To put it simply, our imagery is hyperrealistic. The picture obtained in the preceding step is used to provide face identification. They have selected the Generative Adversarial Network as our model here. A generator and a discriminator are used to teach the model the association. In order to solve the identical problem, they are utilising a context-based Generative Adversarial Network. Once the process of face identification is finished, they use it to identify the individual from the face picture. HOG Face recognition is used for this. They worked to help the police force decrease their time spent on drawing and time spent on criminal identification.

2.1 Problem Statement: Using machine learning technology (von Lilienfeld, 2020), finding relationships and patterns across different data is straightforward specially in big data types. The research in this exploration involves pattern of crime through Machine learning. Also, data visualization helps analyst complex data set. But still exploration in Indian context has been missing.

\section{METHODOLOGY}

Using machine learning technology (von Lilienfeld, 2020), finding relationships and patterns across different data is straightforward specially in big data types. The research in this exploration involves pattern of crime through Machine learning. Also, data visualization helps analyses complex data set. But still exploration in Indian context has been missing.

3.1 Domain overviews: The models can uncover and extract hidden knowledge about crime from a historical criminal data set. The crime pattern prediction model aims to use machine learning techniques on criminal data to aid in the prediction of crime patterns (Sandagiri et al. 2020). With hybrid models and data sets, the proposed models assist in crime pattern prediction and decision-making. This saves money and time in the modelling process, while also improving the accuracy of the results. This suggestion, such as machine learning techniques, appears to be promising as data modelling and analysis tools.

3.2 Existing System/Problem Statements: Major Problem in Review Papers was Not good Accuracy and not God comparison this practice produces unfavorable biases, errors, and a lot of wasted time. Clinical decision support integrated with computer-based criminal records in the proposed system (Data Sets). This reduced medical errors, improve individual safety, reduce unwanted practice variation, and lower crime rates. This suggestion appears to be promising as a tool for data modelling and analysis. This is a social problem because of the exponential penetration of video monitoring Surveillance is yet another part of our present-day lives, and it may be part of this research too.

\subsection{Proposed Methodology}

- Research Design.

- Data Pre-Processing

- Machine Learning, Supervised Learning, Classification Techniques

- Classification, crime pattern (Data Mining Techniques)

- Machine Learning Algorithms

- Significant Feature Selection.

\subsection{Data Pre-Processing}

[A.] Cleaning: Data that we want to process is not be clean, in the sense that it may contain noise or missing values, and if we process it, we don't get good results. To get good and perfect results, we must eliminate all of this, and data cleaning is the process to do so.

[B]. Transformation: This entails transforming data from one format to another in order to make it more understandable by using data normalization, smoothing, and generalization techniques, as well as aggregation techniques.

[c] Integration: Data that we do not need to process may come from multiple sources, and if we do not integrate them, it may cause a problem during processing. As a result, integration is an important phase in data preprocessing, and various issues are considered here to integrate.

[C] Reduction: When working with data, it can be complex and difficult to understand at times. To make them understandable to the system, we reduce them to the required format in order to achieve good results. The subject of the experiment is a set of variables that are kept constant while the subject of the experiment is a set of variables that are measured. This is more practical when conducting face recognition and detection because it monitors a subject's behaviors and patterns to determine whether the subject matches all of the details presented and has been cross-checked with previous data. It is a time-bound method of effect research that focuses on the relationship between the crimes and the criminals who produce actual outcomes.

IV. Data Collection and Preprocessing: This study's data came from the University of California at Irvine's data repository. The data from the UCI and the website Machine Learning Repository is open to the public. Many researchers have found that the Cleveland and Hungarian databases, in particular, are suitable for developing a mining model because they have fewer missing values and outliers. Before being submitted to the proposed algorithm for training and testing, the data is cleaned and preprocessed. The UCI Machine Learning Repository is a collection of databases (Dhankhar \& Jain, 2021), domain theories, and data generators that the machine learning community uses to test machine learning algorithms empirically. The overall goal of our research is to improve our ability to predict the presence of heart disease. To obtain more accurate results, the UCI and repository datasets are used in this paper. 
V. Proposed Techniques: Machine Learning and data analysis techniques that has been used to analyses crime data that has been previously saved from multiple sources in order to identify patterns and trends in crimes in recent years. Such investigations may aid in the speeding up of the criminal investigation process as well as the automated detection of offenders by electronic systems. The relation of crime detection and Machine Learning techniques (Salloum et al. 2020). Ultimately the Crime Detection using Machine Learning depends upon the selection of Selection of Data, Selection of Algorithms and Selection of platform to perform the result.

Figure 1: Crime Detection using Machine Learning

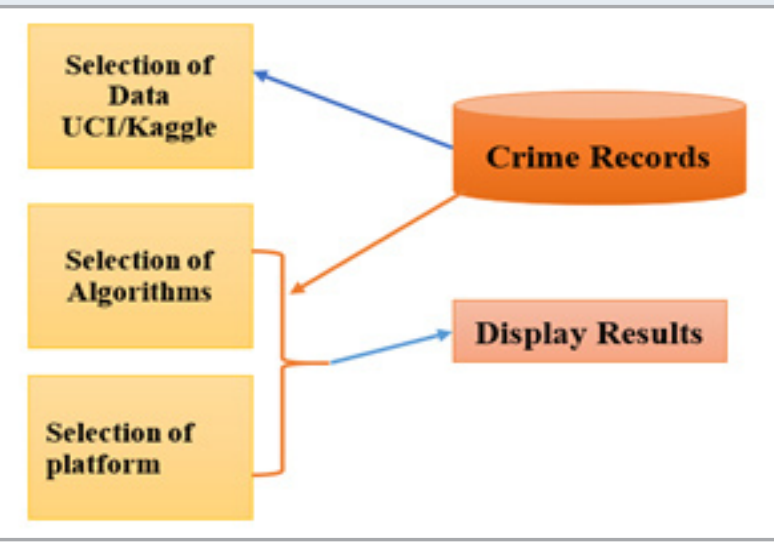

Figure 2: Illustrate the crime detection process using Naïve Bayes

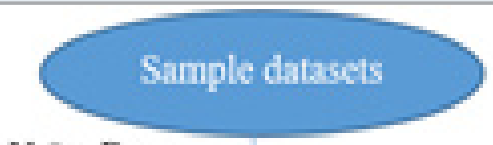

Nalve Bapes

Performanes Comparison

Best Perfom ing Algorithm

Crime and Criminal Database

Chassification Rules

Possible Suspet

5.1 Criminal Identification using Machine Learning: Criminal identification system identifies the suspect of the crime based on the various attributes and the evidences found at the crime scene. To ensure the accuracy of the result, we create a sample dataset and then J48, Naïve Bayes and Machine Learning algorithms be evaluated against it. Their performance compared in reference with accuracy of classification, correctly classified instances, true positive rate, false positive rate, precision, recall etc. The best performing algorithm then selected for actual crime and criminal Machine Learning. So, in general we develop criminal identification system that first classify the sample crime and criminal data in reference with various attributes and generate the classification rules. These classification rules used to identify the possible suspects of the crime. Then details of the suspect be retrieved from the database.

Figure 3: Number of Crimes as per the categories

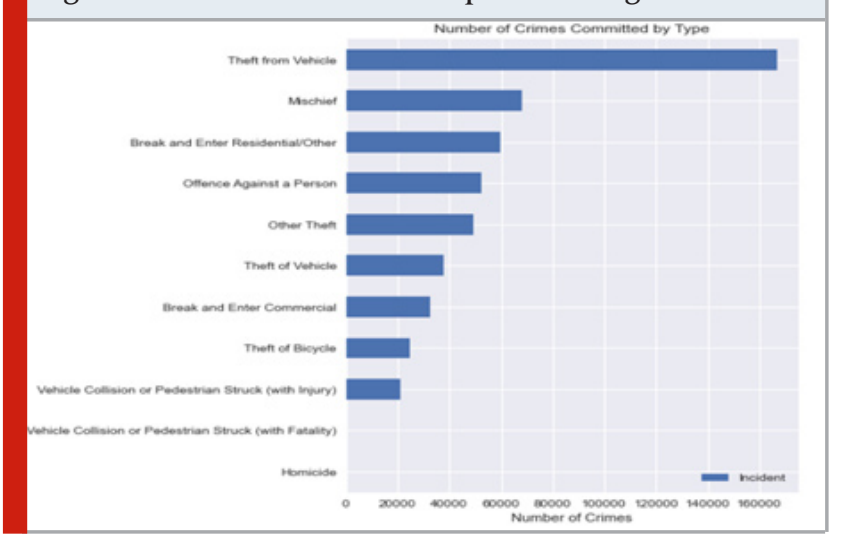

Figure 4: Heat map of the crime data

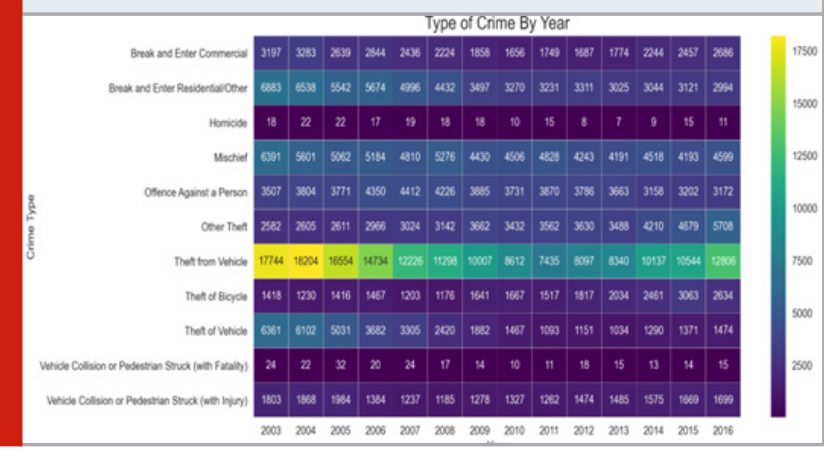

Figure 6: Break and enter commercial

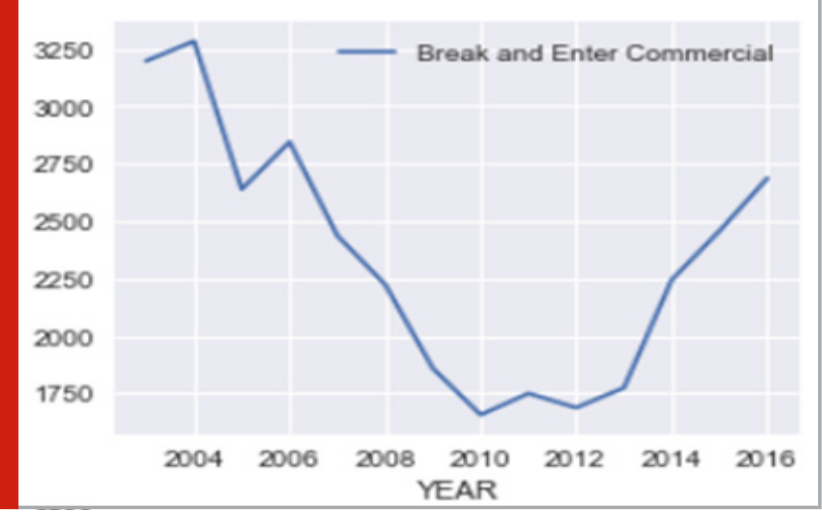

5.2 Decision Tree Based Prediction Model: Decision tree is a compilation of guidelines for studying. Knowledge is split into two or three subsets for allowing inferential analysis. The toughest places to work in would be where people have severe conflicting views. It is split into 
classes until there is just one instance in the leaves. This is achieved using the decision tree classification system. Decision trees are valuable because of their simplicity of discernment and implementation (Han et al., 2008). There is no need to make any pre-processing because the anomaly peaks do not influence the results. Furthermore, the differentiation between persons and groups is well defined. Highlighting is needless in this framework.

Figure 6: Break and Enter Residential/Others

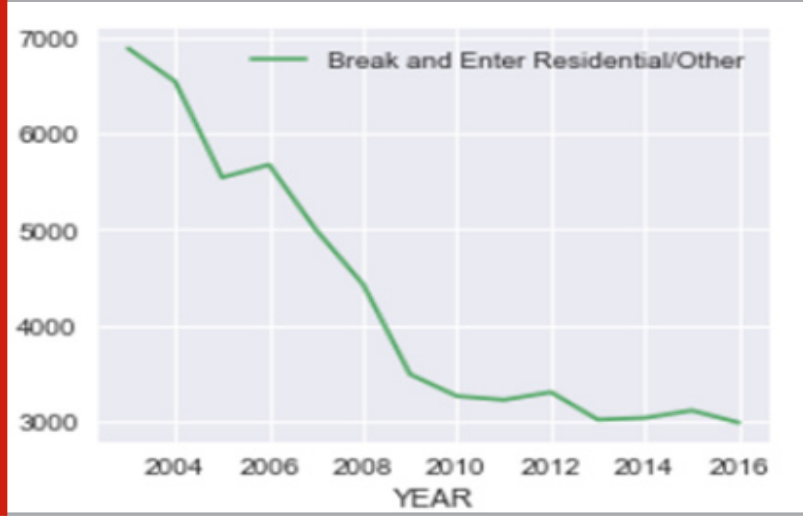

Figure 7: Crime of Homosides

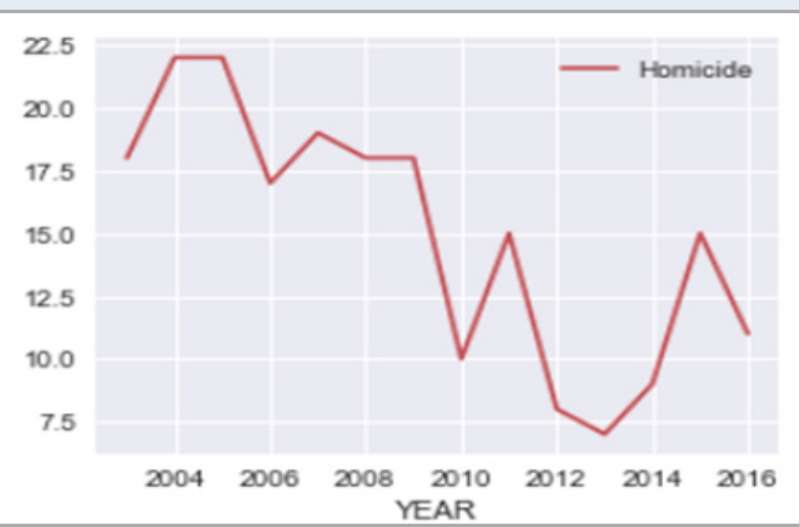

Figure 8: Crime of Homosides

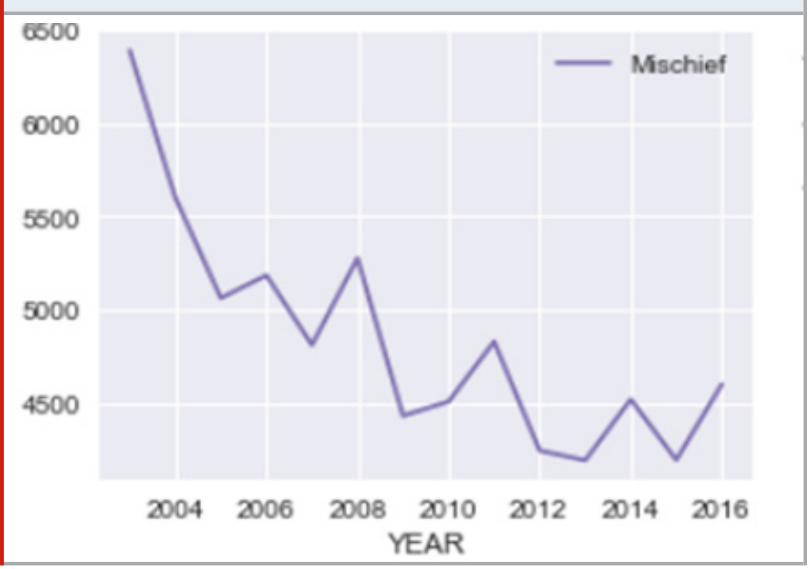

Scaling may produce unexpected effects when the characteristics are personalized. Decision trees succeed at managing discrete quantitative data sets. In this case it muddles the interaction between elements and goals. Decision trees have a higher chance of displaying nonlinear results than linear ones owing to this factor. The decision tree contains many strengths but it still has drawbacks. Firstly, Decision Trees do not suit very well due to unexpected evidence and therefore do not forecast new knowledge well. Trunks of trees cannot be substituted because Decision Trees are starving algorithms.

5.3 Visual Exploration of Data: Execution of the India State wise crime data has been executed in Python. The data sets have the location of Indian crime state wise. Data attributes are Location, Block, Crime Type, Community Area, State wise presentation. Below figure illustrated the categorical attribute distributes.

Figure 9: Offence against a person

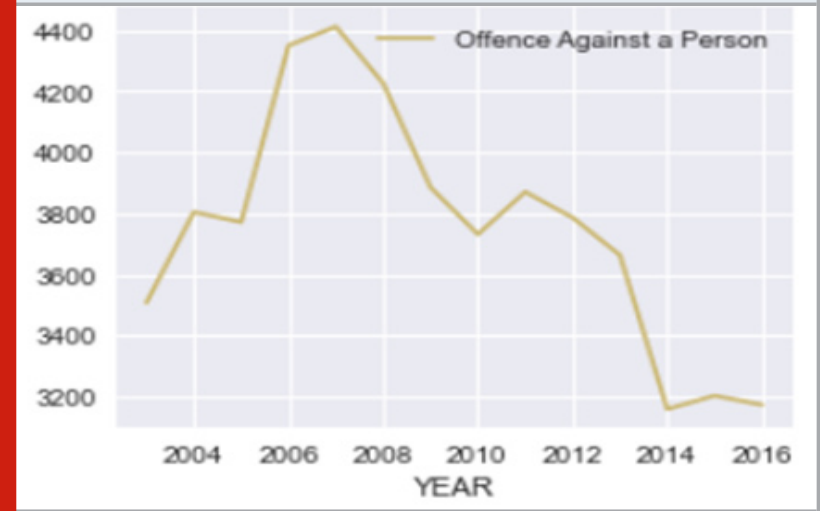

Figure 10: Other Thefts

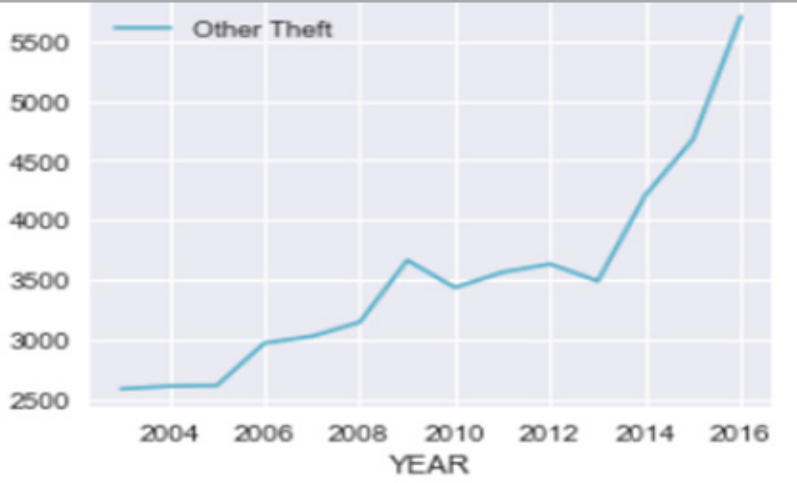

As the figure below also illustrate that the mischief and offence against person. The time series data is from 2004 to 2016 of on record crime data. Heat map of the crime data where it ranges from 2003 to 2016 data. The lighter region belong the most crimed region and the dark side is the lesser crime region. The above figure presented the crime as Break and enter commercial from 2003 from 2016. This figure has two peaks as in 2004 where as the second in 2006 and two slumps as it appeaps in 2010 and 2012. After word it rise continoulsy till 2016. The above figure presented the crime as Break and Enter Residential/Others from 2003 from 2016. This figure has two peaks as in 2004 where as the second in 2006 and 
two slumps as it appeaps in 2013 and 2016. The above figure presented the crime as Homosides from 2003 from 2016. This figure has four peaks as in 2004, 2007,2011 and the last in 2015 and slumps as it appeaps three times as in 2006, 2010 and 2013. The above figure presented the crime as Homosides from 2003 from 2016. This figure has four peaks as in 2006, 2008,2011 and the last in 2014 and five slumps as it appeaps in 2005, 2007, 2009, 2011 and the last in 2015.

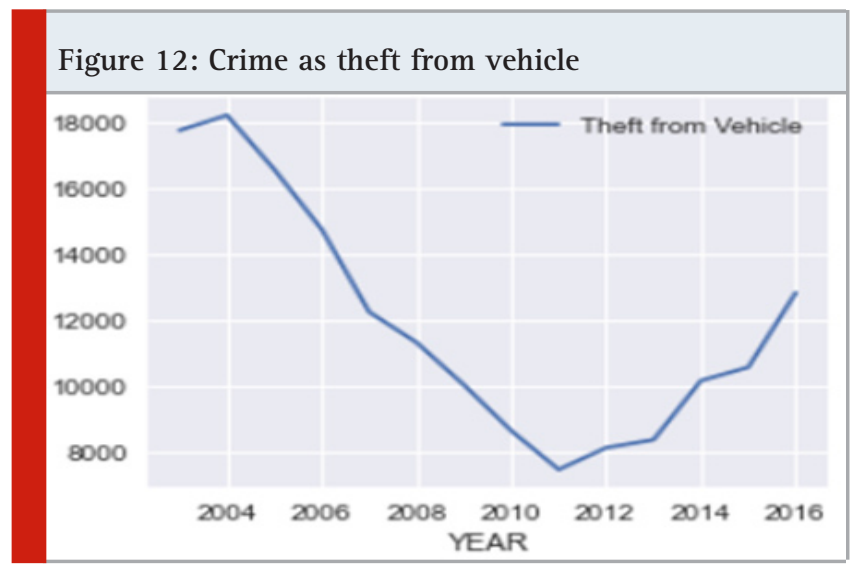

Figure 13: Theft of Bicycles

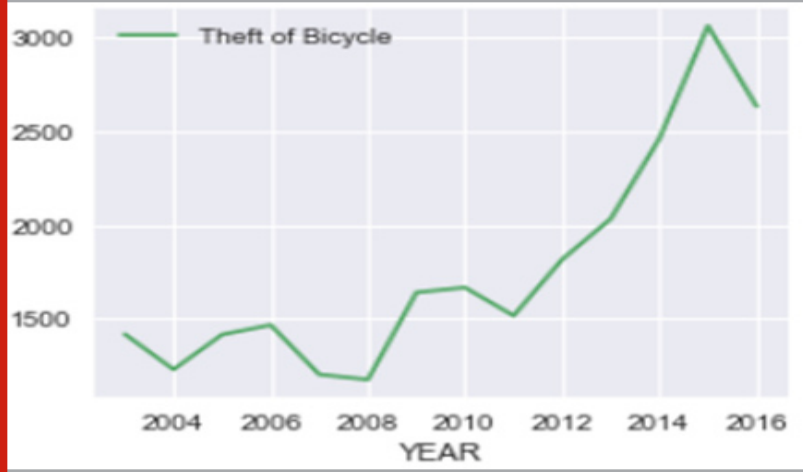

Figure 14: Theft of vehicle

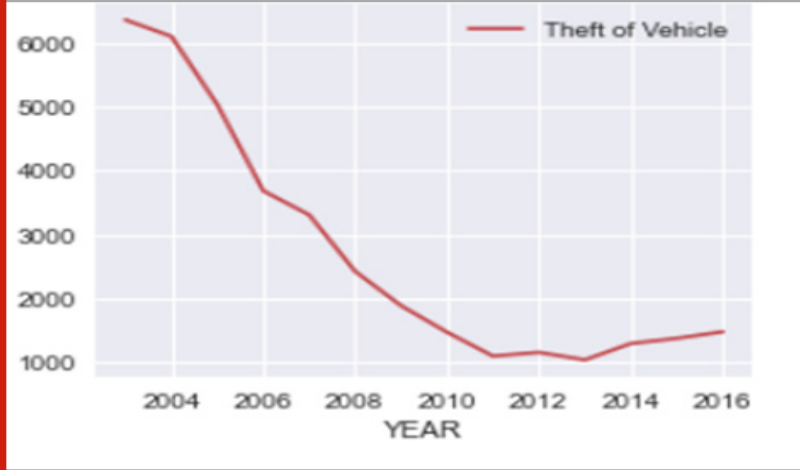

The above figure presented the crime as Offence against a person from 2003 from 2016. This figure has two peaks as in 2007, and the last in 2011 and three slumps as it appeaps in 2005, 2010 and the last in 2014. The above figure presented the crime as Other Thefts from 2003 from 2016. This figure has two peaks as in 2009, and the last in 2016 and one slumps as it appeaps in 2013.
The above figure presented the crime as Other Thefts from 2003 from 2016. This figure has two peaks as in 2009, and the last in 2016 and one slumps as it appeaps in 2013. The above figure presented the crime as Theft of Bicycles from 2004 from 2016. This figure has three peaks as in 2006, 2007 and 2013 and three slumps as it appeaps in 2004, 2008 and 2011. The above figure presented the crime as Theft of Bicycles from 2004 from 2016. This figure has one significant peaks as in 2004 and one significant slump from 2011 to 2013.

Figure 15: vehicle collsion or pedestrian

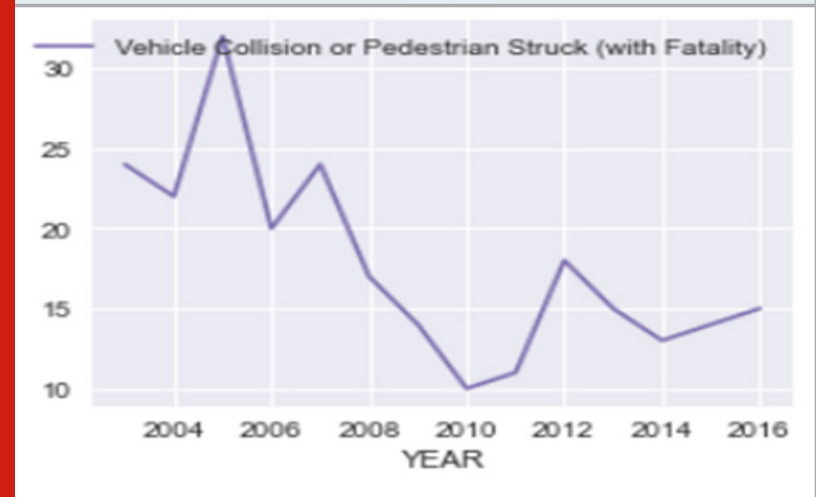

Figure 16: vehicle collsion or pedestrian struck (With Injury)

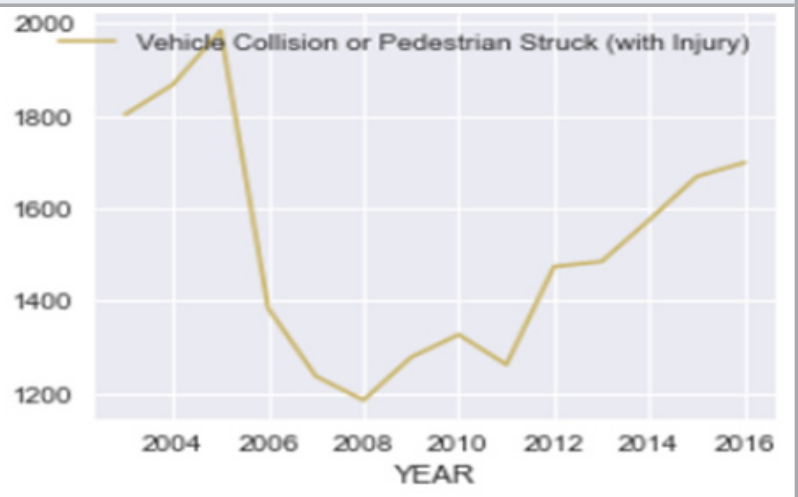

Figure 17: Flow Chart of Prediction using Decision-TreeClassifier (DTC)

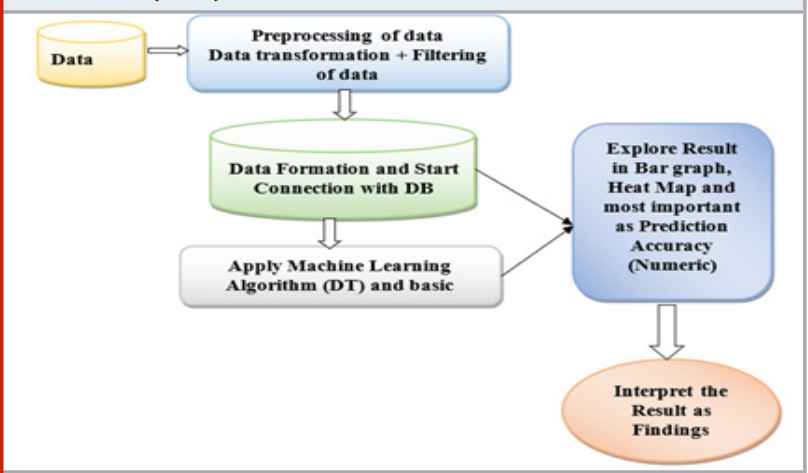

The above figure presented the crime as vehicle collsion or pedestrian struck (With Fatality) from 2004 from 2016. This figure has three peaks as in 2004, 2007 and 2012 and 
four slumps as it appeaps in 2004, 2006, 2010 and 2014. The above figure presented the crime as vehicle collsion or pedestrian struck (With Injury) from 2004 from 2016.
This figure has four peaks as in 2005, 2010,2012 and 2015 and two significant slumps as it appeaps in 2008 and 2011.

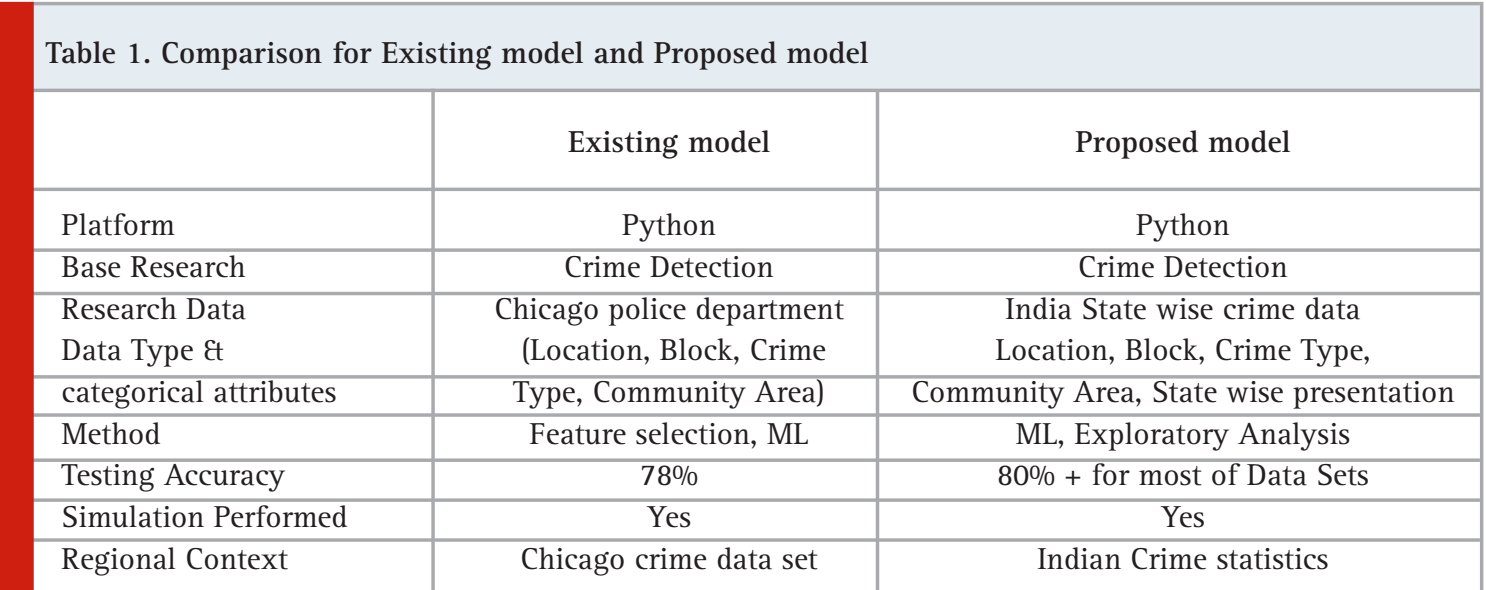

5.4 Prediction using Decision-Tree-Classifier (DTC): As the process to achieve Prediction has been illustration below. This process involves the Decision-Tree-Classifier (DTC) as the prediction algorithm opt form the machine leaning.

Now prediction has been executed through DecisionTree-Classifier (DTC) on the taken data. This data has records of 512849 Which has been broken into 358956 train crime events as tanning purpose and 153839 train crime events for testing purpose as the general traditional ratio of 70/30. Further apply DTC found the accuracy of 43.16 on the given parameter of data.

Findings of the Research: Machine learning algorithms (Decision Tree Classifier) Used to Predict the crime. And exploration of visual data of crime record with simple statistics tools used through Python. A Decision Tree is a straightforward visual model for categorising cases. A Supervised Machine Learning method is one in which the data is continually divided according to a certain parameter. Only Data mining algorithms has been used in most of research and use of the Machine learning is the novelty of this investigation. As this has prediction accuracy of $43 \%$ which is very ethicistic for the inspecting the crimes of very huge data and location. From the existing work this research has distinguish feature of prediction suing the supervised learning algorithm.

\section{CONCLUSION}

The machine learning method is concerned with extracting information or knowledge from huge data sources. It is via inquiry that a large quantity of information is obtained, and only information of value is necessary for analysis. Which therefore demonstrates the ability of Machine Learning to be employed for this purpose. While the choice of Machine Learning algorithm has a bigger impact on the outcome, one of the key reasons for doing comparative analysis and selecting the best performing Machine Learning algorithm is to improve performance. Classifying cases using a Decision Tree is a straightforward concept that everybody can understand. It is a Supervised Machine Learning in which the data is separated according to a given parameter and supervision is constantly applied. Machine learning has only just started to see wider usage in both research and practice, and as a result, the majority of current applications use data mining methods. These particular calculations have an accuracy of above $43 \%$, which is rather impressive when consider that are evaluating crimes from enormous data and locations.

\section{REFERENCES}

Arunima Kumar, Raju Gopal, "Data mining-based crime investigation systems: Taxonomy and relevance”, 2015 Global Conference on Communication Technologies (GCCT) - 2015, DOI: 10.1109/GCCT.2015.7342782

Bachhety, S., Singhal, R., \&t Rawat, K. (2018), Crime Detection Using Text Recognition and Face Recognition. International Journal of Pure and Applied Mathematics, 119(15), 2797-2807.

Baesens, B., Höppner, S., \&t Verdonck, T. (2021). Data engineering for fraud detection. Decision Support Systems, 113492.

Beland, L. P., \&t Brent, D. A. (2018). Traffic and crime. Journal of Public Economics, 160, 96-116.

Chung-Hsien Yu, Max Ward, Melissa Morabito, Wei Ding, "Crime Forecasting Using Data Mining Techniques", 2011 IEEE 11th International Conference on Data Mining Workshops, 2011, DOI: 10.1109/ICDMW.2011.56

David, H.B.F., \&t Suruliandi, A. (2017). Survey on crime analysis and prediction using data mining techniques. ICTACT journal on soft computing, 7(3), 2229-6959.

Dhankhar, A., \&t Jain, S. (2021). Prediction of disease using machine learning algorithms. Smart and Sustainable Intelligent Systems, 115-125.

Dutta, S., Gupta, A. K., \& Narayan, N. (2017, October). Identity crime detection using data mining. In 2017 3rd International Conference on Computational Intelligence 
and Networks (CINE) (pp. 1-5). IEEE.

Han, J., Rodriguez, J. C., \& Beheshti, M. (2008, December). Discovering decision tree-based diabetes prediction model. In International Conference on Advanced Software Engineering and Its Applications (pp. 99-109). Springer, Berlin, Heidelberg.

Hussein, R. R. A., Croock, M. S., \&t Al-Qaraawi, S. M. (2019). Improvement of Criminal Identification by Smart Optimization Method. In MATEC Web of Conferences (Vol. 281, p. 05003). EDP Sciences.

Hussein, R.R.A., \&t Croock, M.S. (2019), Improvement of Criminal Identification by Smart Optimization Method. MATEC Web of Conferences, 2(8).

Hussein, R.R.A., Al-Qaraawi, S.M., \&t Croock, .S. (2020), Viterbi optimization for crime detection and identification. TELKOMNIKA Telecommunication, Computing, Electronics and Control, 18(5), 2378 2384.

Jain, R., Nayyar, A., \&t Bachhety, S. (2020). Factex: A Practical Approach to Crime Detection. In Data Management, Analytics and Innovation (pp. 503-516). Springer, Singapore.

Jayapratha et al. (2018), real time criminology detection and criminal identification (RCDCI). International Journal of Advanced Research in Computer Engineering \&t Technology (IJARCET), 7(5), 2278 - 1323.

Karve, A., Balasubramanian, M., Chaudhari, K., \&t Mane, S. B. (2019, June). Automated Criminal Identification System Using Face Generation. In International Conference on Intelligent Computing, Information and Control Systems (pp. 566-573). Springer, Cham.

Light, M. T., \&t Miller, T. (2018). Does undocumented immigration increase violent crime. Criminology, 56(2), 370-401.

Mittal, M., Goyal, L. M., Sethi, J. K., \&t Hemanth, D. J. (2019). Monitoring the impact of economic crisis on crime in India using machine learning. Computational Economics, 53(4), 1467-1485.
Mittal, M., Goyal, L.M., \& Sethi, J. (2018), Monitoring the Impact of Economic Crisis on Crime in India Using Machine Learning. Comput Econ

Pavithra, R., \&t Suresh, K. V. (2019, April). Fingerprint image identification for crime detection. In 2019 International Conference on Communication and Signal Processing (ICCSP) (pp. 0797-0800). IEEE.

Prabakaran, S., \&t Mitra, S. (2018, April). Survey of analysis of crime detection techniques using data mining and machine learning. In Journal of Physics: Conference Series (Vol. 1000, No. 1, p. 012046). IOP Publishing.

Qayyum, S., \& Dar, H. (2018). A Survey of Data Mining Techniques for Crime Detection. University of Sindh Journal of Information and Communication Technology, 2(1), 1-6.

Salloum, S. A., Alshurideh, M., Elnagar, A., \&t Shaalan, K. (2020). Machine learning and deep learning techniques for cybersecurity: a review. In Joint European-US Workshop on Applications of Invariance in Computer Vision (pp. 50-57). Springer, Cham.

Sandagiri, S. P. C. W., Kumara, B. T. G. S., \& Banujan, K. (2020). Social Media and Online News Analytics for Identifying Crime Patterns in Crime Prediction.

Singh, A. K., Baranwal, N., \&t Nandi, G. C. (2019). A rough set-based reasoning approach for criminal identification. International Journal of Machine Learning and Cybernetics, 10(3), 413-431.

Srivastava, A., Gupta, S., \&t Gupta, K. (2013). Lipstick stain: a silent clue for criminal identification. International Journal of Humanities and Social Science Invention, 2(12).

Tayal, D.K., Jain, A., \& Arora, S. (2015), Crime detection and criminal identification in India using data mining techniques. AI \& Soc, 3(3), 117-127.

Von Lilienfeld, 0. A. (2020). Introducing Machine Learning: Science and Technology. Machine Learning: Science and Technology, 1(1), 010201. 